\title{
Características Mecánicas Dinámicas de la Tibia Aislada de Perro Sometida a Prueba de Impacto
}

\author{
Dynamic Mechanical Characteristics of the Isolated Dog Tibia, Subjected to Impact Test
}

\author{
Fioretti, C."; Galán, A.."; Moine, R."; Varela, M."; Varela, P.**; Mouguelar, H..; \\ Gigena, S.*; Bonino, F.***; Quinteros, R.*** \& Natali, J.*
}

\begin{abstract}
FIORETTI, C.; GALÁN, A.; MOINE, R.; VARELA, M.;VARELA, P.; MOUGUELAR, H.; GIGENA, S.; BONINO, F.; QUINTEROS, R. \& NATALI, J. Características mecánicas dinámicas de la tibia aislada de perro sometida a prueba de impacto. Int. J. Morphol., 31(2):562-569, 2013.
\end{abstract}

RESUMEN: El propósito del presente trabajo es brindar información acerca de las propiedades biomecánicas y morfológicas de la tibia de perro sometida a cargas dinámicas. Los objetivos fueron evaluar la resistencia de la tibia frente al impacto mediante el Método de Ensayo Charpy, relacionar la misma con el área total, el área cortical y el área de la cavidad medular del hueso, comparándola entre talla, edad y sexo diferente. La importancia clínica, ortopédica y quirúrgica que reviste la región de la pierna del perro fundamenta esta investigación. Se utilizaron tibias de perro en condiciones aisladas, extraídas postmortem de 30 animales mestizos adultos jóvenes provenientes del servicio de zoonosis de la ciudad de Río Cuarto. Se determinó en la tibia derecha e izquierda: peso y longitud total del hueso, diámetros cráneo-caudal y latero-medial en la mitad de la diáfisis. A la tibia izquierda se le practicó osteotomía transversal en la mitad de su diáfisis. En la superficie de sección se midió: espesor de la cortical, diámetro medular y se obtuvo el área de sección total, área cortical y área medular. La tibia derecha fue sometida al Método de Ensayo Charpy. Se realizó el análisis estadístico correspondiente. La resistencia del hueso presentó una correlación de $\mathrm{r}=0,70(\mathrm{p}=0,000019) ; \mathrm{r}=0,67(\mathrm{p}=0,000059)$ y $\mathrm{r}=0,29(\mathrm{p}=0,12)$ con respecto a las áreas cortical, total y medular respectivamente. La morfología tiene influencia sobre las propiedades biomecánicas del hueso. A medida que aumenta el área cortical aumenta la energía de impacto absorbida. En cambio, a mayor área de cavidad medular la energía de impacto absorbida aumenta pero en menor magnitud. Existen diferencias estadísticamente significativas $(\mathrm{p}=0,0168)$ entre la talla del animal y la resistencia de la tibia. La edad $(\mathrm{p}=0,73)$ y el sexo $(\mathrm{p}=0,18)$ no presentaron influencias significativas sobre las propiedades biomecánicas de la tibia.

PALABRAS CLAVE: Perro; Tibia; Prueba de Impacto.

\section{INTRODUCCIÓN}

Los huesos, en conjunto con los cartílagos, conforman el esqueleto del animal. Dentro del aparato locomotor constituye la estructura de apoyo del cuerpo, proporcionando su armazón y un sistema de palancas que actúa en la postura y el movimiento (Ruckebusch et al., 1994). Durante la evolución, el esqueleto de los vertebrados desarrolló resistencia a la deformación e indirectamente a la fractura. Se adaptó a los requerimientos de su entorno dentro de los límites fisiológicos de exigencias mecánicas (Currey, 1984; Nordin \& Frankel, 2004). Dichas cualidades se obtuvieron optimizando las dos propiedades determinantes de la resistencia de toda estructura sólida: la calidad mecánica y la distribución espacial del material que la compone (Ferretti et al., 1998; Tascau et al., 2009).
El hueso cortical diafisario es un compuesto material constituido básicamente por hidroxiapatita que le confiere la rigidez y el colágeno que es la proteína responsable de otorgar elasticidad y plasticidad al hueso. La estructura no homogénea, la trabeculación ósea e interposición de fluidos, le proporcionan características de anisotropía y viscoelasticidad al hueso (Cowin et al., 1991; Yeni et al., 1997).

Las características estructurales de los huesos varían según la especie, edad y sexo y se remodela de acuerdo a las necesidades funcionales (Panagiotopoulos et al., 2005; Bediz, 2010). Kokshenev (2007) describe variaciones de los huesos entre especies. Al avanzar la edad, declina la resistencia, la rigidez ósea y la capacidad para absorber energía.

\footnotetext{
* Anatomía Animal, Depto. de Anatomía Animal. Facultad de Agronomía y Veterinaria. Universidad Nacional de Río Cuarto, Argentina.

** Depto. de Mecánica. Facultad de Ingeniería. Universidad Nacional de Río Cuarto, Argentina.

**** Matemáticas y Bioestadística. Facultad de Agronomía y Veterinaria. Universidad Nacional de Río Cuarto, Argentina.

Este trabajo fue subsidiado por Secyt-UNRC (A322)
} 
En el proceso de osteoporosis hay una pérdida de espesor del tejido cortical y trabecular por consecuencia se somete al hueso cortical a una mayor carga fisiológica (Passi \& Gefen, 2005; Syahrom et al., 2011; Green et al., 2011). En este sentido en la actualidad, se están efectuando estudios en tibias de humanos, in vitro e in vivo, con la finalidad de diagnosticar enfermedades metabólicas óseas como la osteoporosis.

La estructura ósea puede estar expuesta a las siguientes fuerzas extrínsecas: axial (compresión o tracción), transversa o deslizamiento, inflexión o flexión y torsión o rotación. Si la magnitud de la aplicación de la carga impuesta no excede la elasticidad límite del hueso no ocurre la fractura y el hueso deformado retorna a su estado anterior. En caso que la energía externa impartida sobrepase la capacidad de almacenaje, ocurre la fractura, dado que el hueso tiene una limitada capacidad de absorción de energía. Entre los factores importantes en la génesis de las fracturas se encuentran la magnitud, duración y dirección de las fuerzas que actúan sobre el hueso (Camargo et al., 2002). Se considera que la resistencia es importante en la biomecánica de los huesos, de ella depende el comportamiento del tejido óseo ante la fractura.

Trabajos sobre resistencia al impacto del fémur de perro mediante el Método de Ensayo Charpy han demostrado que a medida que aumenta el área cortical aumenta la energía de impacto absorbida. Sin embargo, un aumento en el área de cavidad medular, no necesariamente implica una mayor cantidad de energía absorbida (Natali et al., 2008; Fioretti et al., 2011).

Se destaca la poca información disponible sobre aspectos biomecánicos de los huesos de la pierna tanto en humanos como en pequeños animales. Cristofolini et al. (2010), afirman que en el pasado, se han abordado algunos aspectos acerca de la rigidez y la fuerza de los huesos de las extremidades posteriores, pero la información no es suficiente. Mientras que el fémur se ha investigado en mayor medida, hay poca información disponible sobre la tibia y el peroné.

Este trabajo se fundamenta en la importancia clínica, ortopédica y quirúrgica de la región de la pierna del perro, ya que diversos reportes indican que la incidencia de fracturas de tibia en pequeños animales representa el $21 \%$ de las fracturas de huesos largos.

El presente trabajo tiene el propósito de brindar información sobre el comportamiento biomecánico de la tibia de perro sometida a cargas dinámicas y de las características morfológicas del hueso.
Los objetivos fueron evaluar la resistencia de la tibia de perro frente al impacto en la mitad de la diáfisis mediante el Método de Ensayo Charpy y, relacionar la resistencia de la tibia con las áreas: total, cortical y de la cavidad medular del hueso, comparándola entre diferentes talla, edad y sexo.

\section{MATERIAL Y MÉTODO}

Se utilizaron huesos tibias de perro en condiciones aisladas, extraídas postmortem de 30 animales mestizos adultos jóvenes (entre 2 a 10 años) provenientes del servicio de zoonosis de la ciudad de Río Cuarto, Córdoba, Argentina. Debido a la procedencia de dichos animales la edad se determinó por cronología dental.

A los perros se les practicó eutanasia utilizando: Acedán, Tiopental sódico, sangrado y fijación general con formol al 3\%. Se desarticularon ambas tibias y mediante disección convencional se las liberaron de la región. Los procedimientos citados se llevaron a cabo en la cátedra de Anatomía Veterinaria del Departamento de Anatomía Animal de la Facultad de Agronomía y Veterinaria en la Universidad Nacional de Río Cuarto. Este trabajo se llevó a cabo dentro del marco que exige la Comisión de Bioética de la Universidad Nacional de Río Cuarto.

Los perros se agruparon por edad en, grupo a: perros mayor a 3 años y grupo b: perros menor o igual a 3 años y por talla en, grupo 1: talla grande (entre 20 y $30 \mathrm{~kg}$ ) y grupo 2: talla chica (entre 10 y $20 \mathrm{~kg}$ ). Se determinó en ambas tibias (derecha e izquierda): peso, longitud total del hueso, diámetros cráneo-caudal (c-c) y latero-medial (1-m) en la mitad de la diáfisis.

La tibia izquierda fue osteotomizada transversalmente en la mitad de su diáfisis, utilizando para ello una sierra manual. En la superficie de sección se determinó: espesor de la cortical en sus cuadrantes (craneal, caudal, lateral y medial), diámetro medular cráneo-caudal (c-c) y latero-medial (l-m) y se obtuvieron los valores de las áreas de sección total, cortical y medular (Fig. 1). Las medidas fueron tomadas mediante el uso de regla milimétrica de $0,02 \mathrm{~mm}$ de graduación y un microscopio óptico Axiophot (Carl Zeiss, Alemania). Las medidas particulares de la tibia izquierda serán extrapoladas a la tibia derecha contralateral, utilizando la presunción de simetría bilateral que propone Markel et al. (1994). La elección de lateralidad para el hueso osteotomizado responde sólo a un fin metodológico de trabajo.

En forma individual, la mitad de la diáfisis de la tibia derecha fue sometida al Método de Ensayo Charpy (flexión 


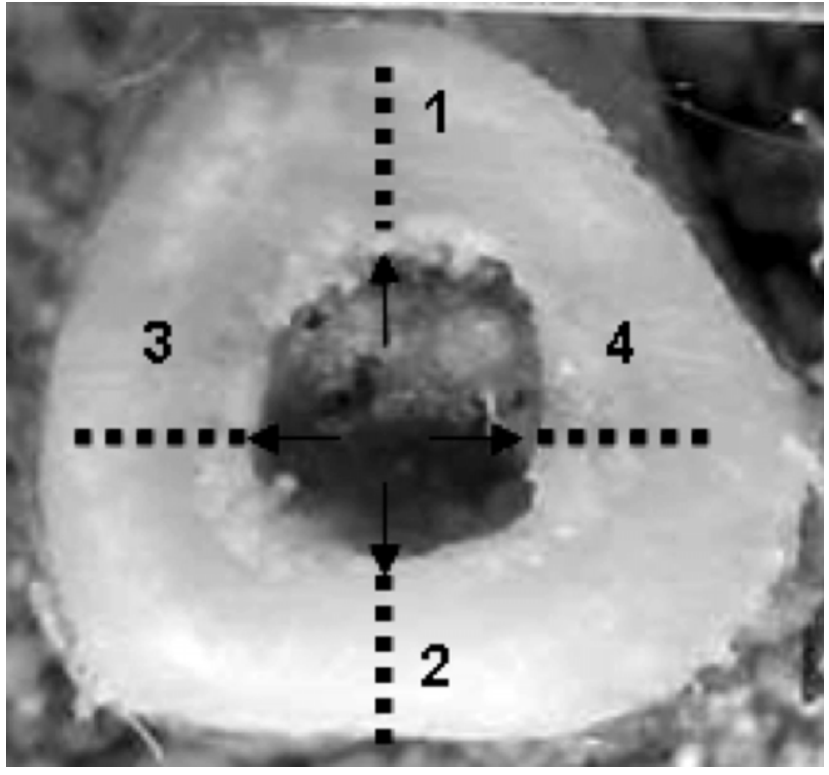

Fig. 1. Corte transversal en la mitad de la diáfisis de la tibia izquierda de perro. Cuadrantes craneal (1), caudal (2), lateral (3), medial (4) de la cortical (Microscopio óptico Axiophot Carl Zeiss).

por impacto). El hueso fue envuelto en gasa humedecida en solución fisiológica y conservado en freezer a $-20 \mathrm{C}^{\circ}$. Previo al ensayo los huesos se descongelaron por método natural y se llevaron a temperatura ambiente. La maquinaria específica para este tipo de ensayo se encuentra en el Laboratorio de Ensayo de Materiales de la Facultad de Ingeniería de la Universidad Nacional de Río Cuarto. El modelo de la Máquina de Ensayos que se usó corresponde a: PW 30/15 $\mathrm{K}-\mathrm{E}$, fabricada por Amsler-Wolpert. El ensayo consiste en golpear la tibia con un péndulo oscilante calibrado, obteniendo como resultado la energía absorbida por el hueso.
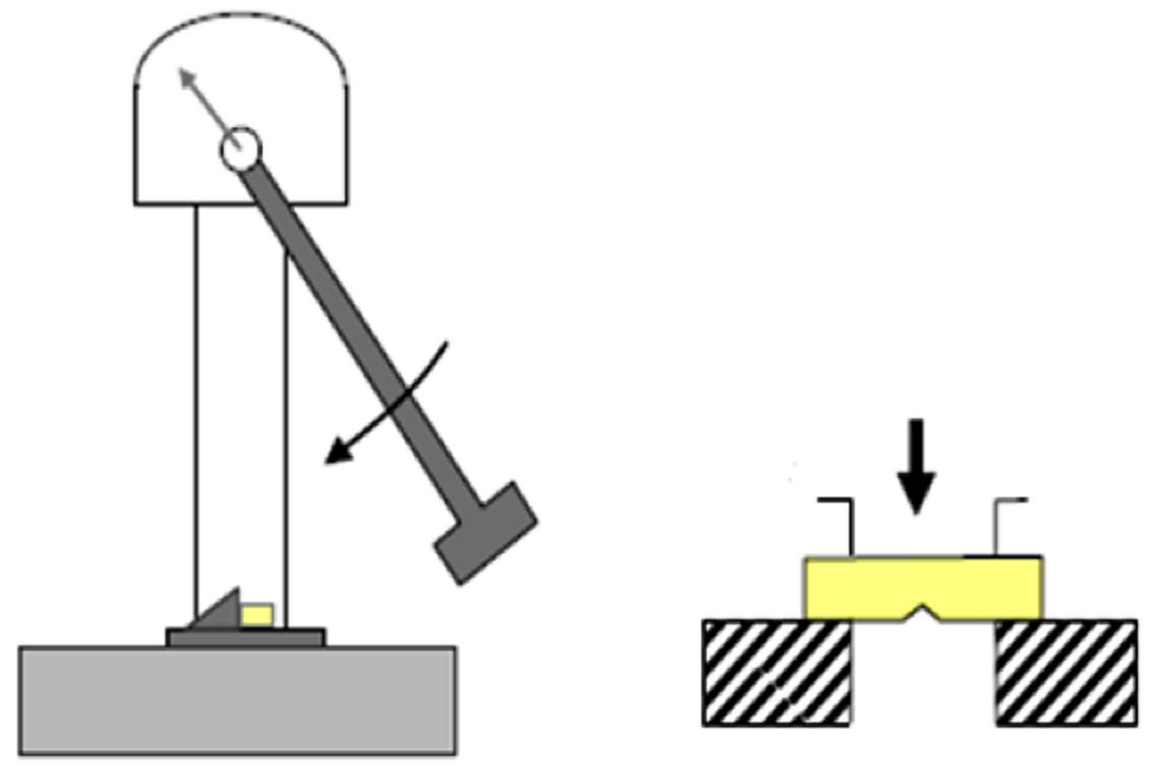

Fig. 2. Esquema de la ubicación del hueso sobre los soportes (Método Charpy). Dirección del impacto (flecha). Tomado de Wang et al. (2010). 


\section{RESULTADOS}

La estadística descriptiva de nuestros datos reflejan (Tabla I), que las variables cuantitativas: peso y longitud

Tabla I. Análisis descriptivo: Medidas resumen de las variables cuantitativas para ambas tibias. Diámetros en la mitad de la diáfisis en $\mathrm{cm}$.

\begin{tabular}{lccccc}
\hline Variables & n & Media & DE & Mínimo & Máximo \\
\hline Peso $(\mathrm{g})$ & 30 & 75,27 & 27,29 & 13,80 & 153,00 \\
Longitud $(\mathrm{cm})$ & 30 & 18,82 & 2,78 & 8,50 & 24,30 \\
Diámetro 1-m & 30 & 1,32 & 0,18 & 0,75 & 1,65 \\
Diámetro c-c & 30 & 1,30 & 0,16 & 0,88 & 1,57 \\
\hline
\end{tabular}

Tabla II. Análisis descriptivo: Medidas resumen para las variables cuantitativas en la mitad de la diáfisis de la tibia izquierda $(\mathrm{cm})$.

\begin{tabular}{lccccc}
\hline Variables & n & Media & DE & Mínimo & Máximo \\
\hline Cortical craneal & 30 & 0,35 & 0,07 & 0,20 & 0,49 \\
Cortical caudal & 30 & 0,35 & 0,06 & 0,23 & 0,46 \\
Cortical lateral & 30 & 0,36 & 0,09 & 0,19 & 0,55 \\
Cortical medial & 30 & 0,36 & 0,07 & 0,23 & 0,50 \\
Cavidad medular c-c & 30 & 0,59 & 0,10 & 0,31 & 0,80 \\
Cavidad medular 1-m & 30 & 0,60 & 0,12 & 0,37 & 0,85 \\
\hline
\end{tabular}

Tabla III. Análisis descriptivo: Medidas resumen para las variables cuantitativas en la mitad de la diáfisis de la tibia izquierda $\left(\mathrm{cm}^{2}\right)$.

\begin{tabular}{lccccc}
\hline Variables & n & Media & DE & Mínimo & Máximo \\
\hline Área total & 30 & 1,36 & 0,33 & 0,55 & 2,11 \\
Área cortical & 30 & 1,08 & 0,29 & 0,45 & 1,77 \\
Área cavidad medular & 30 & 0,28 & 0,10 & 0,10 & 0,47 \\
\hline
\end{tabular}

del hueso; diámetro latero-medial y diámetro cráneo-caudal en la mitad de la diáfisis de la tibia presentaron los siguientes valores promedio: $75,27 \mathrm{~g} ; 18,82 \mathrm{~cm} ; 1,32 \mathrm{~cm}$ y $1,30 \mathrm{~cm}$ respectivamente.

En la Tabla II se muestran las medidas resúmenes y sus desvíos estándar de las variables cuantitativas determinadas en la mitad de la diáfisis de la tibia izquierda: espesor de la cortical (craneal, caudal, lateral y medial), diámetro de la cavidad medular (cráneo-caudal y latero-medial). Se observa que el valor promedio del espesor de la cortical craneal es igual al espesor de la cortical caudal $(0,35 \mathrm{~cm})$. También hay igualdad en el valor promedio entre el espesor de la cortical lateral y medial $(0,36 \mathrm{~cm})$. Los promedios y desvíos estándar para las áreas de la mitad de la diáfisis de la tibia izquierda se muestran en la Tabla III.

La resistencia de la tibia presentó una correlación de $\mathrm{r}=0,70, \mathrm{p}=0,000019$; respecto al área cortical (Fig. 3) y de r=0,67, p=0,000059 respecto al área total (Fig. 4). La correlación entre resistencia y área de la cavidad medular fue de $\mathrm{r}=0,29$, $\mathrm{p}=0,12$.

En la estadística inferencial, se realizaron análisis de correlación para las variables área cortical versus longitud de la tibia y se demostró que el área cortical depende linealmente de la lon-

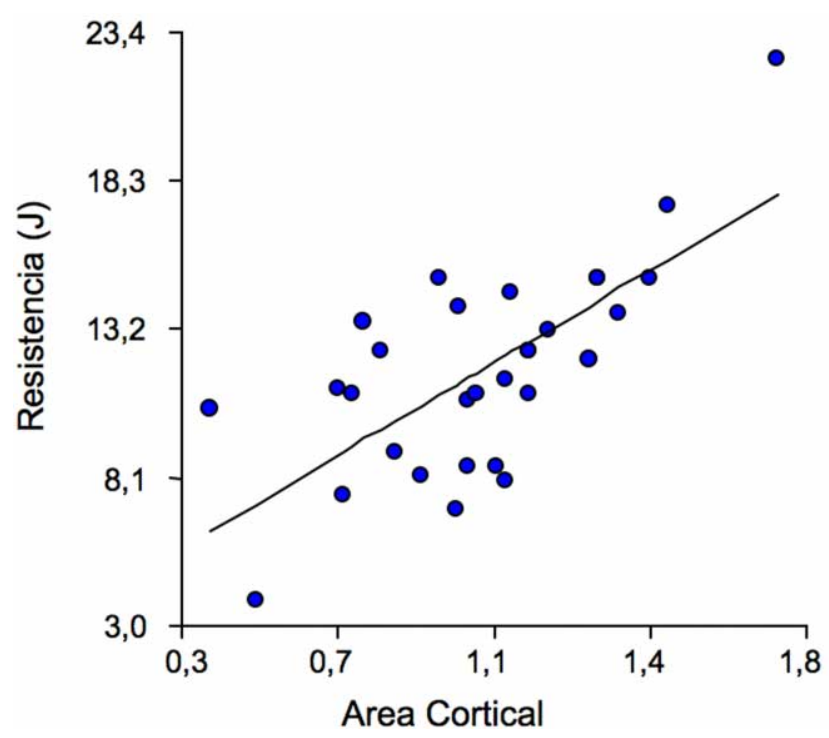

Fig. 3. Recta de regresión: resistencia vs. área cortical.

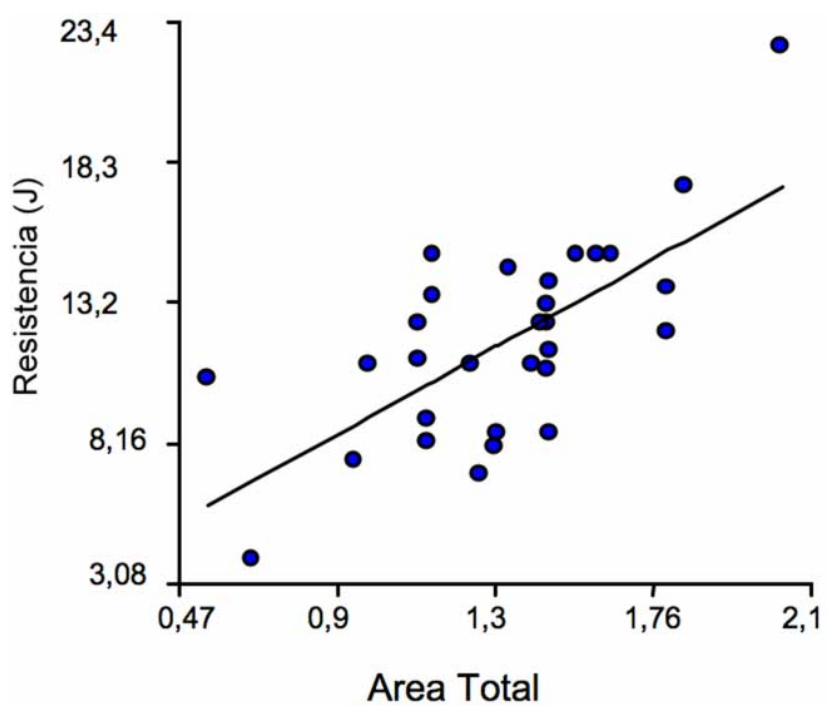

Fig. 4. Recta de regresión: resistencia vs. área total. 
gitud del hueso ( $\mathrm{r}=0,68, \mathrm{p}=0,000036)$, su recta de regresión se muestra en la Figura 5.

La Tabla IV muestra los resultados individuales de los ensayos mecánicos de resistencia y resiliencia (resistencia / área cortical) de la tibia derecha. Los valores obtenidos son expresados en Joule $(\mathrm{J})$ para la resistencia y en $\mathrm{J} / \mathrm{cm} 2$ para la resiliencia. El valor promedio de la resistencia de la tibia fue de 11,57 J (D.E. 4,02) y el valor promedio de la resiliencia fue de $11,29 \mathrm{~J} / \mathrm{cm} 2$ (DE 3,40).

El análisis exploratorio (Fig. 6, box plot de la resistencia versus talla) muestra que existen diferencias signifi- cativas entre la resistencia de la tibia y la talla del perro. Los huesos tibias de animales de talla grande presentaron una mayor resistencia frente al impacto respecto a huesos de animales de talla chica $(\mathrm{p}=0,0168)$.

El análisis exploratorio (Figs. 7 y 8, box plot de la resistencia versus edad y sexo respectivamente) muestran que la edad y el sexo ( $\mathrm{p}=0,73$ y $\mathrm{p}=0,18$ respectivamente) no influyen sobre la resistencia de la tibia, ya que no se encontraron diferencias estadísticas significativas para dichas variables. No obstante, se observa que, en la variable edad los valores de resistencia promedio presentan una mayor dispersión de datos provenientes de las tibias de perros meno-

Tabla IV. Resistencia y resiliencia al impacto en la mitad de la diáfisis de la tibia derecha con sus medias y desvíos estándar $(n=30)$.

\begin{tabular}{lccccc}
\hline n Perro & $\begin{array}{c}\text { Resistencia tibia } \\
(\mathbf{J})\end{array}$ & $\begin{array}{c}\text { Resiliencia tibia } \\
\left(\mathbf{J} / \mathbf{c m}^{\mathbf{2}}\right.\end{array}$ & $\mathbf{n}$ Perro & $\begin{array}{c}\text { Resistencia tibia } \\
(\mathbf{J})\end{array}$ & $\begin{array}{c}\text { Resiliencia tibia } \\
\left(\mathbf{J} / \mathbf{c m}^{\mathbf{2}}\right)\end{array}$ \\
\hline 1 & 12,50 & 10,50 & 16 & 7,50 & 9,87 \\
2 & 11,00 & 9,90 & 17 & 14,00 & 13,60 \\
3 & 15,00 & 10,20 & 18 & 13,20 & 10,64 \\
4 & 10,80 & 10,28 & 19 & 15,00 & 11,11 \\
5 & 15,00 & 11,11 & 20 & 4,00 & 7,14 \\
6 & 12,50 & 14,70 & 21 & 11,00 & 14,10 \\
7 & 13,80 & 9,85 & 22 & 7,00 & 6,86 \\
8 & 17,50 & 11,58 & 23 & 2,00 & 14,93 \\
9 & 15,00 & 15,30 & 24 & 12,50 & 8,09 \\
10 & 9,00 & 10,22 & 25 & 8,50 & 7,01 \\
11 & 8,20 & 8,72 & 26 & 8,00 & 10,08 \\
12 & 11,00 & 10,28 & 27 & 11,50 & 12,71 \\
13 & 14,50 & 12,60 & 28 & 22,50 & 23,33 \\
14 & 12,20 & 9,17 & 29 & 10,50 & 7,59 \\
15 & 13,50 & 16,60 & 30 & 8,50 & \\
\hline
\end{tabular}

Media: Resistencia (1,57) y Resiliencia (11,29); DE: Resistencia (4,02) y Resiliencia $(3,40)$.

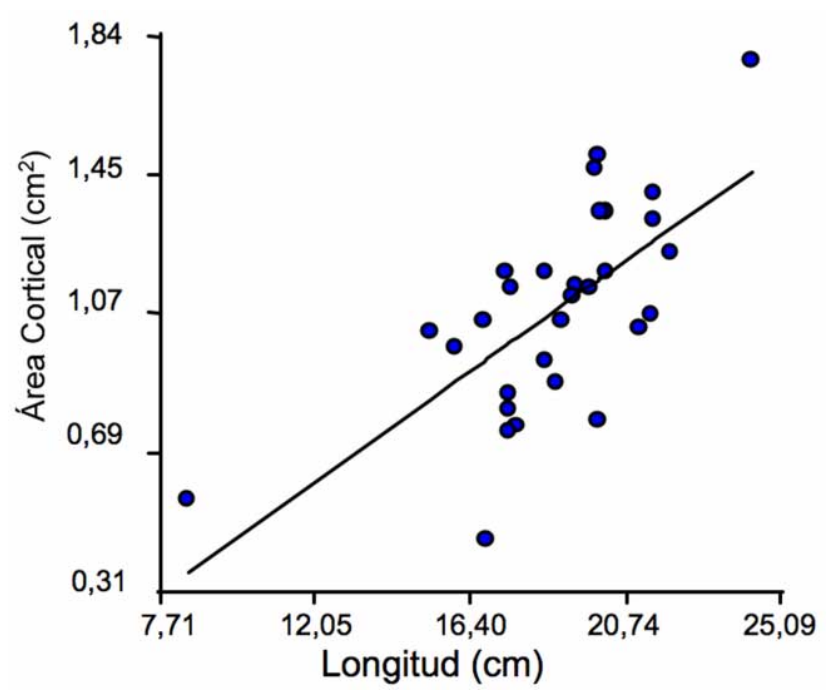

Fig. 5. Recta de regresión: área cortical vs. longitud del hueso.

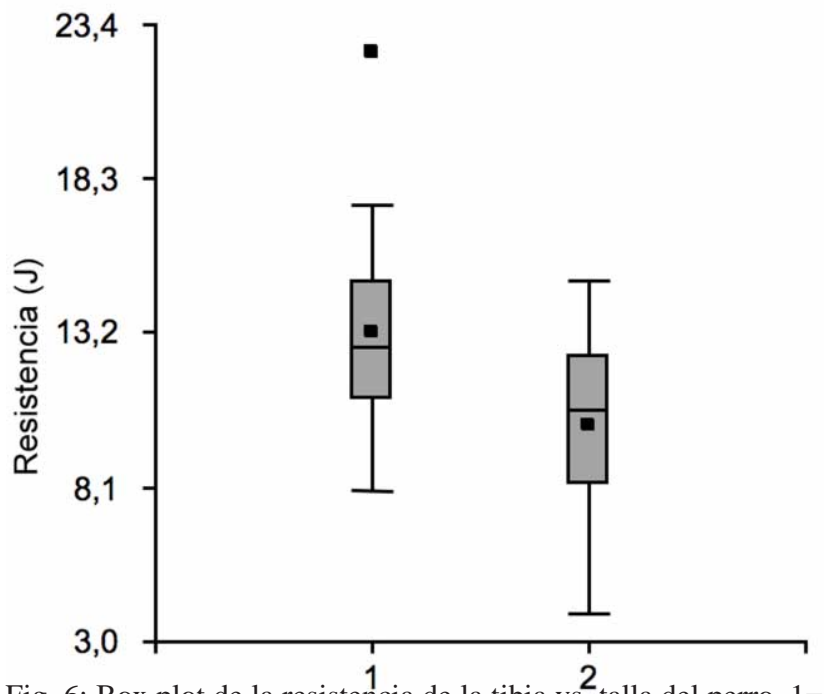

Fig. 6: Box plot de la resistencia de la tibia vs. talla del perro. $1=$ talla grande; $2=$ talla chica 
res o iguales a 3 años. Se observa también que, las tibias de perros machos presentaron una resistencia levemente superior que aquellas de hembras.
Las Tablas V, VI y VII muestran los análisis de la varianza para la resistencia de la tibia versus talla, edad y sexo del perro, respectivamente.
Tabla V. Análisis de la Varianza: resistencia de la tibia versus talla (SC tipo III).

\begin{tabular}{lccccc}
\hline F.V. & SC & gl & CM & F & p-valor \\
\hline Modelo & 70,36 & 1 & 70,36 & 6,47 & 0,0168 \\
Talla & 70,36 & 1 & 70,36 & 6,47 & 0,0168 \\
Error & 304,45 & 28 & 10,87 & & \\
Total & 374,81 & 29 & & & \\
\hline
\end{tabular}

Tabla VI. Análisis de la Varianza: resistencia de la tibia versus edad (SC tipo III).

\begin{tabular}{lccccc}
\hline F.V. & SC & gl & CM & F & p-valor \\
\hline Modelo & 1,57 & 1 & 1,57 & 0,12 & 0,7343 \\
Edad & 1,57 & 1 & 1,57 & 0,12 & 0,7343 \\
Error & 373,24 & 28 & 13,33 & & \\
Total & 374,81 & 29 & & & \\
\hline
\end{tabular}

Tabla VII. Análisis de la Varianza: resistencia de la tibia versus sexo (SC tipo III).

\begin{tabular}{lccccc}
\hline F.V. & SC & gl & CM & F & p-valor \\
\hline Modelo & 22,70 & 1 & 22,70 & 1,81 & 0,1898 \\
Sexo & 22,70 & 1 & 22,70 & 1,81 & 0,1898 \\
Error & 352,10 & 28 & 12,58 & & \\
Total & 374,81 & 29 & & & \\
\hline
\end{tabular}

\section{DISCUSIÓN}

La tibia derecha se sometió al ensayo mecánico bajo temperatura ambiente constante $\left(25^{\circ} \mathrm{C}\right) \mathrm{y}$ utilizando la misma maquinaria de impacto para toda la muestra.

En coincidencia con Yeni et al., Syahrom et al., y Green et al., en este trabajo se comprobó que la morfología de la tibia tiene mucha importancia frente a la resistencia de del hueso a la fractura. Estudios sobre fémur y tibia de humanos destacaron que los parámetros morfológicos pueden explicar, en gran medida, la influencia que ejercen dichos parámetros en la variabilidad de resistencia que presenta el hueso. Coincidimos con Turner \& Burr (1993) en que la fuerza externa requerida para fracturar un hueso es diferente a la resistencia intrínseca del hueso, por lo que dicha fuerza varía acorde a las medidas del hueso. Currey determinó que el aumento de la cortical se puede atribuir a la adaptación del hueso a las cargas de tensión y compresión que ejercen sobre él, esto también prepararía a la cortical a resistir impacto.

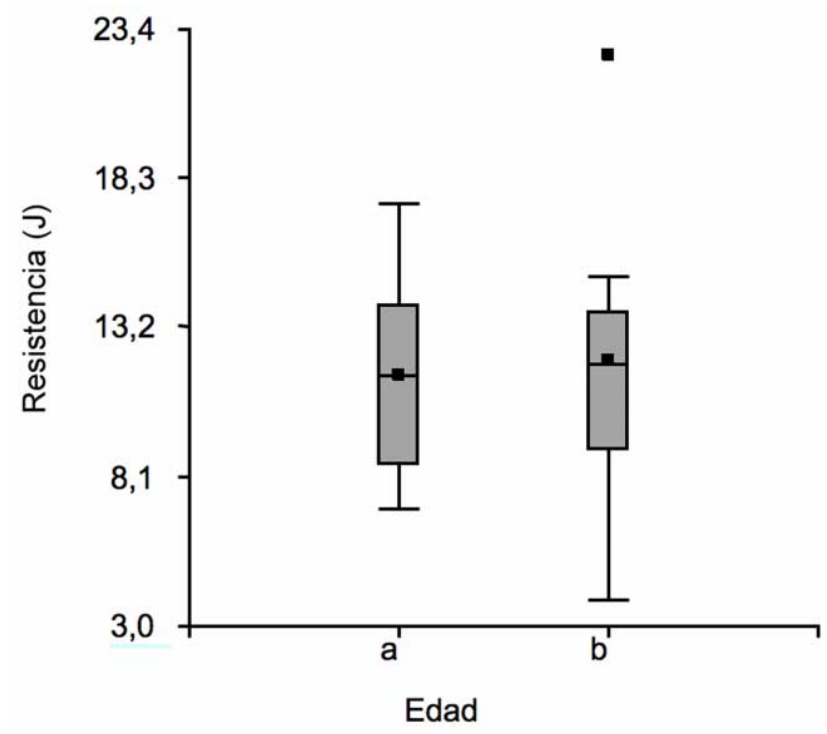

Fig. 7. Box plot de la resistencia de la tibia vs. edad del perro. $a=>$ a 3años; $b=\leq 3$ años.

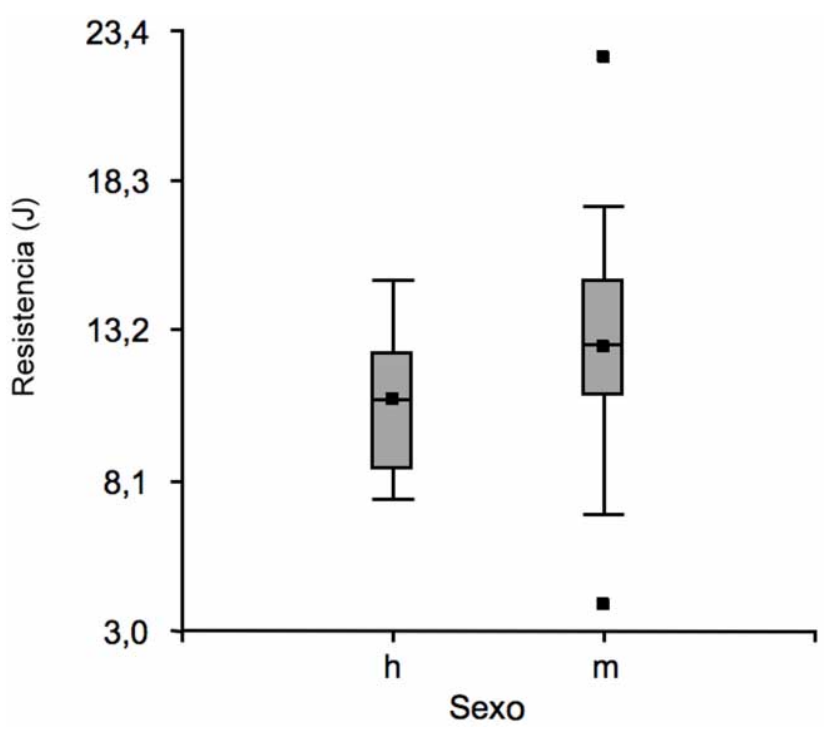

Fig. 8. Box plot de la resistencia de la tibia vs. sexo del perro. 
La resistencia promedio del hueso tibia fue de $11,57 \mathrm{~J}$ $\pm 4,02$ y la resiliencia fue de $11,29 \pm 3,40$. Cuando se toma en cuenta la resiliencia, observamos la menor dispersión de los datos debido a que se toma en cuenta el área cortical en el punto de impacto.

Tomando como ejemplo un determinado animal (n 28 de nuestra muestra) de talla grande, observamos que con una longitud de $24,3 \mathrm{~cm}$ y un área cortical de $1,77 \mathrm{~cm}^{2}$ resistió al impacto 22,5 J. En cambio otro animal (n 20) de talla chica, con una longitud de $8,5 \mathrm{~cm}$ y un área cortical de $0,56 \mathrm{~cm}^{2}$ resistió al impacto $4 \mathrm{~J}$. Es evidente que al prevalecer la longitud total del hueso, el espesor y área cortical en el sitio de impacto, influencian de manera directa con una mayor resistencia del hueso. En este sentido y concordando con nuestros resultados, Wang et al. (2010), expresa que para los huesos largos el tamaño y la forma del hueso constituyen factores relevantes en su funcionamiento biomecánico. Natali et al. y Fioretti et al., también observaron que los huesos en los cuales prevalece el área cortical responden con una mayor resistencia. Coincidimos entonces, en la importancia que ejerce el tamaño del área cortical en el momento de evaluar la resistencia del hueso.

La variable talla del animal, influye en la capacidad de resistencia de la tibia. Se comprobó en este trabajo que hay diferencias estadísticamente significativas entre la resistencia de las tibias del grupo 1 (talla grande) con la resistencia presentada por las tibias del grupo 2 (talla chica). El valor promedio de resistencia al impacto fue de $13,25 \mathrm{~J}$ y $9 \mathrm{~J}$ para las tibias de perros del grupo 1 y grupo 2 respectivamente con un $\mathrm{p}=$ 0,0168 .

La edad avanzada se asocia con una declinación en la resistencia, rigidez y capacidad del hueso para absorber energía. Nuestros resultados muestran que el grupo de animales menores o iguales a 3 años no presentaron diferencias significativas $(p=0,73)$ en su resistencia al impacto, con respecto a aquellos mayores de 3 años. El grupo de animales mayores de 3 años presentó la mayor dispersión de datos. Proviniendo los huesos de animales adultos, los resultados que se obtuvieron en este trabajo fueron los esperados.

Tomando en cuenta la variable sexo, nuestros datos no demuestran evidencias estadísticamente significativas $(p=0,18)$ como para afirmar que existe influencia del sexo sobre la resistencia de la tibia, si bien se observa en el box plot que los perros machos presentaron una resistencia media mayor que las hembras. Esto último concuerda con Panagiotopoulos et al., quien consideró al sexo como factor que puede influir en la resistencia ósea a la ruptura. En jóvenes, la variable sexo tiene importancia significativa en el momento de evaluar las cualidades biomecánicas del tejido óseo, mientras que en grupos de mayor edad la influencia por sexo es menor. Los hue- sos fémur de hombres jóvenes son más resistentes al impacto que los mismos en mujeres jóvenes. El envejecimiento parece reducir estas diferencias, por lo cual es interesante conocer que los huesos de los ancianos tienen la misma absorción de energía independiente del sexo.

La resistencia de la tibia presentó un coeficiente de correlación muestral de 0,70, 0,67 y 0,29 respecto al área cortical, área total y área de la cavidad medular respectivamente. Estos resultados coinciden con Tascau et al., quienes afirman que la cavidad medular de la tibia de perro no tendría incidencia significativa sobre los parámetros mecánicos del hueso.

Finalmente, se coincide con Nordin \& Frankel, Heiner (2008) y Bediz (2010) quienes observaron que la capacidad del tejido óseo no se agota sólo en las cualidades de absorción de impactos, sino que posee un alto nivel de adaptación a los estímulos recibidos. Además como tejido vivo y dinámico, el hueso puede reorganizar sus elementos y adquirir una estructura que se ajusta permanentemente a las demandas de factores mecánicos que actúan sobre él.

En conclusión, existen diferencias de resistencia al impacto entre tibias de perros de diferentes tallas frente a la aplicación del Método de Ensayo Charpy. Los huesos de perros de talla grande resistieron más que los de talla chica. Las variables edad y sexo no presentaron influencias estadísticamente significativas en la resistencia al impacto del hueso tibia. Las variables geométricas tienen gran influencia sobre la resistencia al impacto en la mitad de la diáfisis de la tibia, entre ellas, el tamaño del área cortical es la de mayor influencia. El funcionamiento biomecánico de los huesos largos está condicionado por sus propiedades estructurales y materiales y por la carga impuesta.

FIORETTI, C.; GALÁN, A.; MOINE, R.; VARELA, M.; VARELA, P.; MOUGUELAR, H.; GIGENA, S.; BONINO, F.; QUINTEROS, R. \& NATALI, J. Dynamic mechanical characteristics of the isolated dog tibia, subjected to impact test. Int. J. Morphol, 31(2):562-569, 2013.

SUMMARY: The purpose of this work is to provide information about biomechanical and morphological properties of the dog tibia bone subject to dynamic loads. The objectives were: evaluate the resistance of the tibia subject impact used Charpy test Method; linking it with total area, cortical area and bone marrow cavity; and compare it with weight, age and sex categories. The clinical, orthopedical and surgical relevance of the dog leg region is the basis for this research. Dog tibia were used in isolated conditions, removed postmortem from 30 adult animals defined raceless, from the zoonoses service of the city of Río Cuarto. Weight and total bone length, cranial-caudal and lateral-medial diameter in the middle of the diaphysis, were determined in the left and right tibia. The left tibia was osteotomized transversely in the middle of the diaphysis. On the surface section we measured: cortical thickness, diameter of the bone marrow cavity, and obtained the total section area, cortical area and medullary area. The right tibia was subjected to Charpy test method. 
Statistical analysis was performed accordingly. The bone strength showed a correlation of $r=0.70(p=0.000019) ; r=0.67(p=0.000059)$ and $r=0.29$ $(\mathrm{p}=0.12)$ with respect to the cortical area, total area and medullary area respectively. The morphology has influence on the biomechanical properties of bone, with increasing cortical area increases the impact energy absorbed. In contrast, a greater area of the medullary cavity of impact energy absorbed increases but to a lesser magnitude. There were statistically significant differences $(\mathrm{p}=0.0168)$ of size on the strength of the tibia. Age ( $\mathrm{p}=0.7343)$ and sex $(\mathrm{p}=0.1898)$ had no significant influence on the biomechanical properties of the tibia.

KEY WORDS: Dog; Tibia; Impact test.

\section{REFERENCIAS BIBLIOGRÁFICAS}

Bediz, B. Measuring Structural Dynamic Properties of Human Tibia by Modal Testing. Biomech. Clin., 25(4):159-65, 2010.

Camargo O. P.; Martins, P.; Andrade, R. M.; Duran, C. E. P.; Croci, A. T.; Leivas, T. P.; Pereira, C. A. M. \& Neto, R. B. Comparative analysis of dog femur resistance after receiving circular and square holes. Acta Ortop. Bras., 10(2):41-7, 2002.

Cowin, S. C.; Moss-Salentijn, L. \& Moss, M. L. Candidates for the mechanosensory system in bone. J. Biomech. Eng., 113(2):191-7, 1991.

Cristofolini, L.; Conti, G.; Juszczyk, M.; Cremonini, S.; Van Sint Jan, S. \& Viceconti, M. Structural behaviour and strain distribution of the long bones of the human lower limbs. J. Biomech., 43(5):826$35,2010$.

Currey, J. D. The mechanical properties of materials and the estructure of bone. In: The Mechanical Adaptation of Bone. Princeton, University Press, 1984. pp.3-37.

Ferretti, J. L. Biomechanical properties of bones. En: Genant, H.; Guglielmi, G.; Jergas, M. (Eds.). Bone Densitometry and Osteoporosis. Berlín, Springer, 1998. pp.143-61.

Fioretti, C.; Natali, J.; Galán, A.; Rivera, M. C.; Moine, R.; Varela, P.; Varela, M.; Bonino, F. \& Quinteros, R. Características Mecánicas Dinámicas del Fémur Aislado de Perro, Sometido a Prueba de Impacto. Int. J. Morphol., 29(3):716-22, 2011.

Green, J. O.; Wang, J.; Diab, T.; Vidakovic, B. \& Guldberg, R. E. Agerelated differences in the morphology of microdamage propagation in trabecular bone. J. Biomech., 44(15):2659-66, 2011.

Heiner, A. D. Structural properties of fourth-generation composite femurs and tibias. J. Biomech., 41(15):3282-4, 2008.

Kokshenev, V. B. New insights into long-bone biomechanics: are limb safety factors invariable across mammalian species? J. Biomech., 40(13):2911-8, 2007

Markel M. D.; Sielman, E.; Rapoff, A. J. \& Kohles, S. S. Mechanical properties of the long bones in dogs. Am. J. Ve.t Res., 55(8):117883, 1994.
Natali, J.; Wheeler, J. T.; Kohl, R. \& Varela, P. Comparación de las Características Mecánicas Estáticas del Fémur Aislado de Perro, con y sin la Colocación de una Placa de Ortopedia Fabricada en Polipropileno. Int. J. Morphol., 26(4):791-7, 2008.

Nordin, M. \& Frankel, V. H. Biomecánica básica del sistema musculoesquelético. $3^{\mathrm{a}}$ ed. Madrid, McGraw-Hill Interamericana. 2004. pp.5-150. Cap. 1 у 2.

Panagiotopoulos, E.; Kostopoulos, V.; Tsantzalis, S.; Fortis, A. P. \& Doulalas, A. Impact energy absorption by specimens from the upper end of the human femur. Injury, 36(5):613-7, 2005.

Passi, N. \& Gefen, A. Trabecular bone contributes to strength of the proximal femur under mediolateral impact in the avian. J. Biomech. Eng., 127(1):198-203, 2005

Ruckebusch, Y.; Phaneuf, L. P. \& Dunlop, R. Fisiología de pequeñas y grandes especies. México D.F., El Manual Moderno, 1994. pp.5726.

Syahrom, A.; Kadir, M. R. A. \& Muslim, D. A. J. Relationship between morphological parameters of cancellous bone and its mechanical properties. Proceedings of 2010 IEEE EMBS Conference on Biomedical Engineering and Sciences, IECBES, 2010. pp.336340

Tascau, T.; Dreucean, M. \& Rusu, L. Biomechanical behavior of canine tibia based on bending tests and numerical analysis. 5th International Symposium on Applied Computational Intelligence and Informatics, 2009. pp.129-132.

Turner, C. R. \& Burr, D. B. Basic biomechanical measurements of bone: a tutorial. Bone, 14(4):595-608, 1993.

Wang, X.; Nyman, J.; Dong, X.; Leng, H. \& Reyes, M. Fundamental Biomechanics in Bone Tissue Engineering. Synthesis Lectures on Tissue Engineering. LaVergne, Morgan \& Claypool Publishers, 2010. pp.59-62. Cap. 3 y 4.

Yeni, Y. N.; Brown, C. U.; Wang, Z. \& Norman, T. L. The influence of bone morphology on fracture toughness of the human femur and tibia. Bone, 21(5):453-9, 1997.

\section{Dirección para correspondencia: \\ José Natali \\ Anatomía Animal \\ Departamento de Anatomía Animal \\ Facultad de Agronomía y Veterinaria \\ Universidad Nacional de Río Cuarto \\ Campus Universitario. (5800) Río Cuarto, Cba \\ ARGENTINA}

Email: jnatali@ayv.unrc.edu.ar

Recibido : 08-10-2012

Aceptado: 04-03-2013 